\title{
The role of nucleotide composition in premature termination codon recognition
}

Fouad Zahdeh ${ }^{1,2}$ and Liran Carmel ${ }^{1 *}$ (D)

\begin{abstract}
Background: It is not fully understood how a termination codon is recognized as premature (PTC) by the nonsense-mediated decay (NMD) machinery. This is particularly true for transcripts lacking an exon junction complex (EJC) along their $3^{\prime}$ untranslated region (3'UTR), and thus degrade through the EJC-independent NMD pathway.

Results: Here, we analyzed data of transcript stability change following NMD repression and identified over 200 EJC-independent NMD-targets. We examined many features characterizing these transcripts, and compared them to NMD-insensitive transcripts, as well as to a group of transcripts that are destabilized following NMD repression (destabilized transcripts).

Conclusions: We found that none of the known NMD-triggering features, such as the presence of upstream open reading frames, significantly characterizes EJC-independent NMD-targets. Instead, we saw that NMD-targets are strongly enriched with $\mathrm{G}$ nucleotides upstream of the termination codon, and even more so along their $3^{\prime} U T R$. We suggest that high $\mathrm{G}$ content around the termination codon impedes translation termination as a result of mRNA folding, thus triggering NMD. We also suggest that high $\mathrm{G}$ content in the $3^{\prime} U T R$ helps to activate NMD by allowing for the accumulation of UPF1, or other NMD-promoting proteins, along the $3^{\prime} U T R$.
\end{abstract}

Keywords: Nonsense-mediated decay (NMD), EJC-independent NMD, NMD-triggering features, Stop codon GC content, Stop codon nucleotide composition, RNA secondary structure, Exon junction complex (EJC), Transcription termination

\section{Background}

Nonsense-mediated decay (NMD) is a major eukaryotic surveillance mechanism that targets for degradation transcripts that harbor a premature termination codon (PTC). A primary role of this mechanism is in posttranscriptional quality control, preventing the formation of truncated proteins that are potentially detrimental to the cell $[1-5]$. In addition, NMD is often combined with alternative splicing to form an important regulatory program of gene expression [6-9]. Altogether, NMD is central to maintaining normal cellular activity, and disruption of its proper function is estimated to be associated with about one third of inherited genetic disorders, as well as with many forms of cancer [10-12]. However, the decision-making process at the basis of NMD,

\footnotetext{
* Correspondence: liran.carmel@huji.ac.il

${ }^{1}$ Department of Genetics, The Alexander Silberman Institute of Life Sciences, Faculty of Science, The Hebrew University of Jerusalem, Edmond J. Safra Campus, Givat Ram, Jerusalem 91904, Israel

Full list of author information is available at the end of the article
}

determining whether a termination codon (TC) is premature or not, is still not fully understood.

It is generally believed that the context of the TC within the transcript determines whether it is normal or premature, but the nature of the contextual signals is unclear. The leading hypothesis in mammals is that a $\mathrm{TC}$ is recognized as premature if the pre-mRNA harbors an intron more than 50-55 bases downstream of the TC $[13,14]$. The splicing reaction usually leaves traces in the form of a protein complex called the exon junction complex (EJC), which is deposited upon the mRNA 20-24 bases upstream of the exon-exon junction $[15,16]$. It is believed that the NMD-triggering feature is the presence of EJCs in the 3' untranslated region (3'UTR) at the time of translation termination. The underlying mechanistic explanation is that the first ribosome that scans the transcript clears EJCs from the coding sequence (CDS), but is unable to clear EJCs that are downstream enough to the TC [17] (hereinafter, 3'UTR EJCs). This model explains why the presence of a translated upstream open reading 
frame (tuORF) is also known to trigger NMD [18-21], as the downstream CDS likely harbors EJCs which behave, in the context of the tuORF, as 3'UTR EJCs. In fact, it was suggested that many of the NMD-targets result from short tuORFs, leading to an enrichment of transcripts with short CDS among NMD-targets [22], explaining why short CDS was also reported as NMD-triggering feature in yeast [22] and Drosophila [23].

Despite overall support, this model of PTC recognition is incomplete, as in various cases it was reported to be violated: some transcripts trigger NMD despite of not harboring 3'UTR EJCs [24-27]_ENREF_20, while others are known to evade NMD despite of presenting 3'UTR EJCs [24, 28-30] or tuORFs [31]. These exceptions brought about the idea that NMD works through several different pathways, and led to a distinction between EJC-dependent NMD - which is the NMD that degrades transcripts that harbor 3'UTR EJCs - and EJC-independent NMD, or failsafe NMD - which is the NMD that works on transcripts that lack 3'UTR EJCs [32].

An alternative model (or, more precisely, a set of similar models), known as the faux-UTR model, suggests that a normal transcript is characterized by certain signals in its 3'UTR, and that disruption of these signals triggers NMD [33]_ENREF_24. Substantial evidence supports the notion that one of the central signals that marks a transcript as normal is physical proximity between the TC and the poly(A) binding protein PABPC1 $[2,34-37]$, and that a TC is tagged as premature by the lack of such physical proximity. Presumably, in a normal transcript the translation termination factor eRF3 binds PABPC1, whereas anything that promotes NMD, like an EJC situated downstream of the TC, competes with the PABPC1 and allows eRF3-bound up-frameshift protein 1 (UPF1) to trigger NMD. This model not only clarifies the part that 3'UTR EJCs play in NMD, but also explains why very long 3'UTRs are also known to trigger NMD $[38,39]$, as the TC and PABPC 1 are separated by a very long physical distance. However, as for other NMDtriggering features, a long 3'UTR does not always result in NMD $[36,40]$.

These models assume that UPF1, on which NMD critically depends, works through specific binding with the translation termination complex [41, 42]_ENREF_37. Further evidence, however, points to the possibility that UPF1 is not only recruited by the terminating ribosome, but is associated directly with the mRNA, and is even thought to have helicase activity allowing it to slide along it [43]. It is still debated whether this association is translation-dependent [44-46] or independent [47], but all agree that UPF1 is displaced from the CDS during translation, leading to its enrichment along the 3'UTR $[20,48]$. Opinions also diverge regarding the question whether NMD-targets have excess of UPF1 molecules bound to their 3'UTR. Some report that UPF1 density along the 3'UTR is higher in NMD-targets [45, 49]_ENREF_40. Others do not see significant difference in UPF1 densities between NMD-targets and NMDinsensitive transcripts, claiming that it is not the UPF1 density that matters for NMD, but rather the density of the activated (phosphorylated) UPF1 [44].

NMD may be directly linked to the efficiency of translation termination. It was proposed that unfavorable context of the TC may affect the recruitment of terminating factors, leading to changes in the kinetics of translation termination, and eventually to ribosome stalling at the TC that is thought to be the event triggering NMD $[48,50,51]$. Combined with the presence of 3'UTRbound UPF1, this led to the suggestion that prolonged translation termination allows more UPF1 molecules to bind to the 3'UTR and become activated via regulated phosphorylation, resulting in activation of NMD $[44,48,51]$ ENREF_43.

One of the biggest challenges in understanding PTC recognition stems from the observation that neither of the proposed NMD-triggering features characterizes a large fraction of NMD-targets. Even when all features are considered together, it was estimated that they characterize roughly $30 \%$ of NMD-targets [20]. This suggests that there may be many more yet unidentified features that are important for PTC recognition. Surprisingly, the nucleotide composition around the TC has not received much attention. Hurt et al. found that UPF1 tends to bind 3'UTRs in G-rich regions, possibly because of pausing of UPF1 scanning in these regions [20]. The same authors also reported that NMD-insensitive transcripts with long 3'UTRs are enriched in poly(A) stretches, perhaps because those stretches recruit PABPC1, thus marking the TC as normal [20]. Studies on codon usage bias show that codons near the TC tend to be AT-rich [52-54], conceivably as a result of selection against the formation of RNA secondary structures near the TC that may interfere with the recruitments of release factors and lead to improper translation termination. Yet, the nucleotide composition of the sequence upstream the stop codon in the coding region was never regarded as a potential NMD triggering feature.

Here, we wished to identify additional features that are involved in the decision-making process of NMD. For this, we have used available genome-wide data on RNA stability following UPF1 knockdown [55], and identified transcripts that are targets for degradation. Specifically, we focused on transcripts that lack 3'UTR EJCs, and therefore our targets degrade through the EJC-independent NMD. For each transcript we have computed a long list of features, and tested their relevance to degradation by evaluating whether any of them shows a unique distribution 
within NMD-targets, when compared to non-targets. The features we have tested include all those that had been previously described as associated with NMD, as well as many new ones, mainly measuring nucleotide composition around the TC.

We found that NMD-targets are characterized by high G content around the TC and throughout the 3'UTR. However, we noticed a group of transcripts that are not NMD-targets and yet demonstrate the same, albeit weaker, pattern of enrichment. Interestingly, these transcripts are not NMD-insensitive either, but show decreased mRNA stability following UPF1 knockdown, and are thus called 'destabilized'. These transcripts might show instability due to factors other than NMD. Surprisingly, the 3'UTR length is similar for NMDtargets and NMD-insensitive transcripts, but it is substantially shorter for the destabilized transcripts. Based on these observations we suggest that high G content around the TC and throughout the 3'UTR leads to aberrant translation termination that triggers NMD. We show that this model has a far greater discriminative power than any other EJC-independent NMD-triggering feature known today.

\section{Results}

\section{Identifying NMD-targets}

The ability to identify transcripts that are NMD-targets is a critical starting point in any analysis of NMDtriggering features. NMD-targets are traditionally identified as transcripts whose characteristic behavior is affected by knocking-down a core NMD component. The most common approach is to mark as NMD-targets transcripts whose expression level is increased following UPF1-knockdown. However, this approach is expected to have a high rate of false positives due to transcripts that are secondarily up-regulated. Moreover, the false negative rate may also be high due to genuine NMDtargets whose expression level is stabilized due to feedback regulation [56]. Recently, a different approach was introduced by Tani et al. [55], who used 5'-bromo-uridine immunoprecipitation chase-deep sequencing (BRICseq) at four time points to measure genome-wide mRNA stability. They compared control to UPF1-knockdown samples, and marked as NMD-targets transcripts whose half-life was significantly increased following UPF1knockdown. While this approach may still suffer from secondary effects, it is nonetheless a more direct measure of RNA stability.

The current analysis starts with the raw data of Tani et al. [55]. We have followed parts of their analysis pipeline, but developed a novel method to detect NMDtargets. Tani et al. estimated the decay rate of each transcript by modeling its mRNA abundance decay curve. However, decay curves of NMD-targets often deviate from a simple exponential model, reflecting a mixtures of currently degrading transcripts with older transcripts that had escaped degradation [57]. Given that Tani et al. only used four time points, their half-life estimates especially when the decay is not a simple exponentmay have large standard errors. To circumvent this, we did not attempt to estimate decay rates, and instead developed a test that compares the shapes of the mRNA abundance decay curves and marks as NMD-targets transcripts whose decay curve following UPF1-knockdown is significantly above their control decay curve (Fig. 1a). This group of transcripts is denoted 'stabilized'.

Theoretically, knocking down UPF1 should result in stability increase of NMD-targets, and stability stasis for NMD-insensitive transcripts. However, by using the technique above in the reverse direction we found transcripts whose stability decreases upon UPF1-knockdown, namely, their mRNA abundance decay curves following UPF1knockdown are significantly below their control decay curves (Fig. 1b). Such transcripts are unexpected by current theory, and are denoted here 'destabilized'. Transcripts whose decay curves did not change significantly

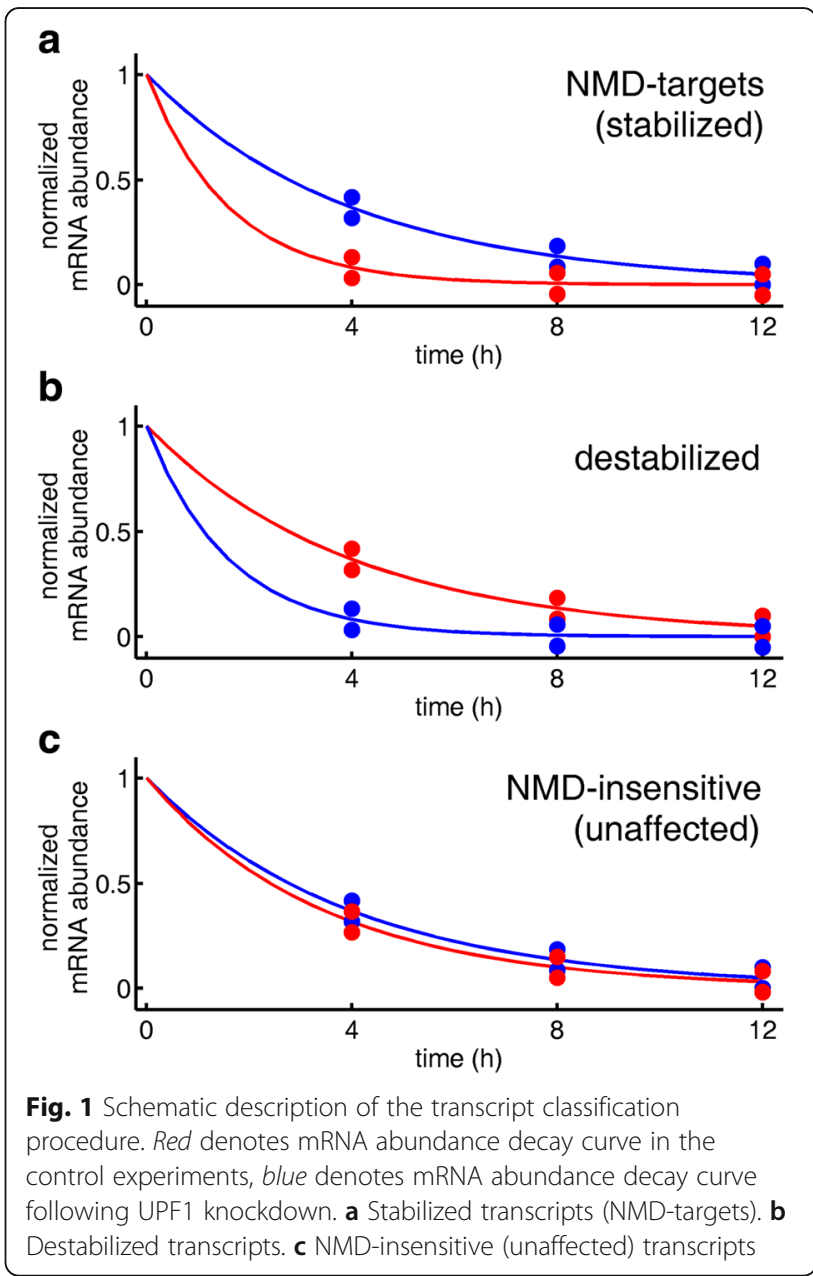


following UPF1-knockdown were marked as 'unaffected', and represent transcripts that are not targeted for degradation by NMD (Fig. 1c). Finally, transcripts whose decay curves showed inconsistent behavior were left unclassified and were excluded from further analysis.

We have re-analyzed the data of Tani et al. and obtained mRNA abundance information for a total of 6080 transcripts. From these, we removed 11 whose mRNA abundance seemed to increase with time. Of the remaining 6069 transcripts, $217(3.6 \%)$ were labeled as stabilized (NMD-targets), $169(2.8 \%)$ were labeled as destabilized, $4329(71.3 \%)$ were labeled as unaffected, and 1354 (22.3\%) were left unclassified. In total, we could classify 4715 transcripts for which we carried out all subsequent analyses. To test the robustness of our results, we have repeated the process using a different, stricter, classification criterion totaling in 4016 classifiable transcripts (Additional file 1: Table S1).

\section{Generating a set of NMD-triggering features}

For each of the 4715 classified transcripts we computed the following features, which were all previously suggested as characterizing NMD-targets in mammals:

- tuorf. A binary feature, indicating the presence/ absence of translated uORFs strictly within the 5'UTR.

- 3'UTR length, in bases.

- 3'UTR A and G content. A and G density at the entire sequence of the $3^{\prime} \mathrm{UTR}$.

- CDS length, in bases.

- $A L U$. A binary feature, indicating the presence/ absence of ALU elements in the 3'UTR. These elements are not directly associated with NMD, but are nevertheless thought to be involved in mRNA degradation through Staufen-mediated decay [58] (see Discussion).

- ALU density. The density of ALU elements within the 3'UTR.

We did not consider 3'UTR EJC presence/absence as a feature, because the transcript list used by Tani et al. was based on the RefSeq database, which normally excludes transcripts that harbor 3'UTR EJCs [19]. Indeed, our data include only 14 transcripts with 3'UTR EJCs. Ten of them were classified as unaffected, whereas the other four were left unclassified. This may reflect a bias in RefSeq annotations towards the inclusion of 3'UTR EJC-harboring transcripts only if confirmed as resistant to NMD. Therefore, the transcripts we have identified in this work as NMD-target are targets of EJC-independent NMD.

These features explain just part of the differences between NMD-targets and transcripts that are not marked for degradation [20]. In order to better characterize NMD-targets, we have examined many more features, none had been previously reported to be associated with NMD. Most of these features capture various aspects of the nucleotide composition around the TC. These features are:

- $s E J C$. A binary feature, indicating the presence/ absence of a 'shallow-EJC', meaning an EJC that comes from an intron that resides in a 3'UTR, but less than 55 bases downstream of the TC.

- tovORF. A binary feature, indicating whether a tuORF overlaps the main ORF.

- Mononucleotide content. All four nucleotide densities at the entire sequence of the 3'UTR, as well as at the last 20 bases of the CDS.

- Dinucleotide content. All 16 dinucleotide densities at the entire sequence of the 3'UTR, as well as at the last 20 bases of the CDS.

- Nucleotide runs: The number of nucleotide runs (three or more consecutive identical nucleotides) at the entire sequence of the 3'UTR, as well as at the last 20 bases of the CDS. This number was compared to the number expected by chance based on the nucleotide densities, and the resulting $x^{2}$-statistics were also used as features.

We ended up with a list of 63 different features. It is expected that this set of features contains many interdependencies, and we have therefore removed features that are highly correlated with other features. This had left us with a set of 43 non-redundant features, of which four are binaries (Additional file 1: Table S2).

\section{Instable transcripts are characterized by high GC content around the termination codon}

We next wished to find which features display a different distribution in stabilized transcripts (NMD-targets) when compared to unaffected ones. For the non-binary features we used the Mann-Whitney $U$-test, whereas for the binary features we used the Fisher exact test. In both cases the $p$-values were FDR-corrected. None of the binary features was found to be significantly different between the two groups of transcripts (Additional file 2: Figure S1A, Additional file 1: Table S3), but many non-binary features did. However, we noticed that the actual difference in the distributions between the two groups is typically very small. In order to account for this, we computed for each non-binary feature the non-parametric common language effect size [59]. For a feature measured for samples coming from two groups $C_{1}$ and $C_{2}$, the effect size $A$ is defined as the probability that the feature value for a random sample from $C_{1}$ would be higher than its value for a random sample from $C_{2}$. Therefore, the closer $A$ is to 0.5 , the 
weaker is the effect. In total, 11 features were found to be both significantly different between stabilized and unaffected transcripts $(P<0.05)$ and to have high effect size $(|A-0.5| \geq 0.1)$ (Fig. 2a, Additional file 1: Tables S2 and S4). Interestingly, all of them describe the nucleotide composition around the TC. At the 3'UTR side they include G content, several dinucleotide contents (AG, GA, CT, and TG), the number of G-runs, and the $\chi^{2}$ G-run statistic. At the CDS side they include $C$ content, and several dinucleotide contents (GG, AT and TA). All these features point at higher GC content upstream to a PTC as well along the entire 3'UTR (Fig. 3). Differences in 3'UTR G content between stabilized and unaffected transcripts are compatible with a recent study that found a preference for G in UPF1 binding sites at the 3'UTR of putative NMDtargets [20]. However, binding of UPF1 is unlikely to be the reason for this enrichment, as high G content extends also to the CDS, and significant enrichment is also observed for C's.

We have repeated the above analysis to compare destabilized transcripts with unaffected ones, and found a total of six non-binary features that are significantly different and with high effect size (Additional file 1: Tables S2 and S4). Two of them (3'UTR G and CT contents) are also the features that are most discriminative between stabilized transcripts and unaffected ones (Additional file 1: Table S2). More generally, all these non-binary features show a similar trend of high GC content around the TC for stabilized and destabilized transcripts. This suggests that this characterizes transcripts that are more prone to stability modifications, regardless of the nature of the specific degradation pathway.

\section{NMD-targets are characterized by high $\mathrm{G}$ content around the termination codon}

Although stabilized and destabilized transcripts show similar nucleotide composition around the TC, we noticed that some features behave differently in the two groups (Fig. 2a-b). In order to test whether any of the features discriminates directly between stabilized and destabilized transcripts, we have carried out the above analysis for a third time, now comparing destabilized transcripts with stabilized ones. We found that four such features, all related to 3'UTR G content (3'UTR G, AG, GA contents and 3'UTR G runs; Fig. 2c). This suggests that NMD-targets have higher $\mathrm{G}$ content around the TC, particularly along the 3'UTR. Supporting our observation that $G$ content is elevated on both sides of the TC, we note that the dinucleotide GG is significantly enriched upstream to the TC of stabilized transcripts, but not in destabilized ones.

Among the binary features, the only one that significantly separates destabilized transcripts from unaffected ones is the presence of ALU elements in the 3'UTR (Additional file 2: Figure S1A, Additional file 1: Table

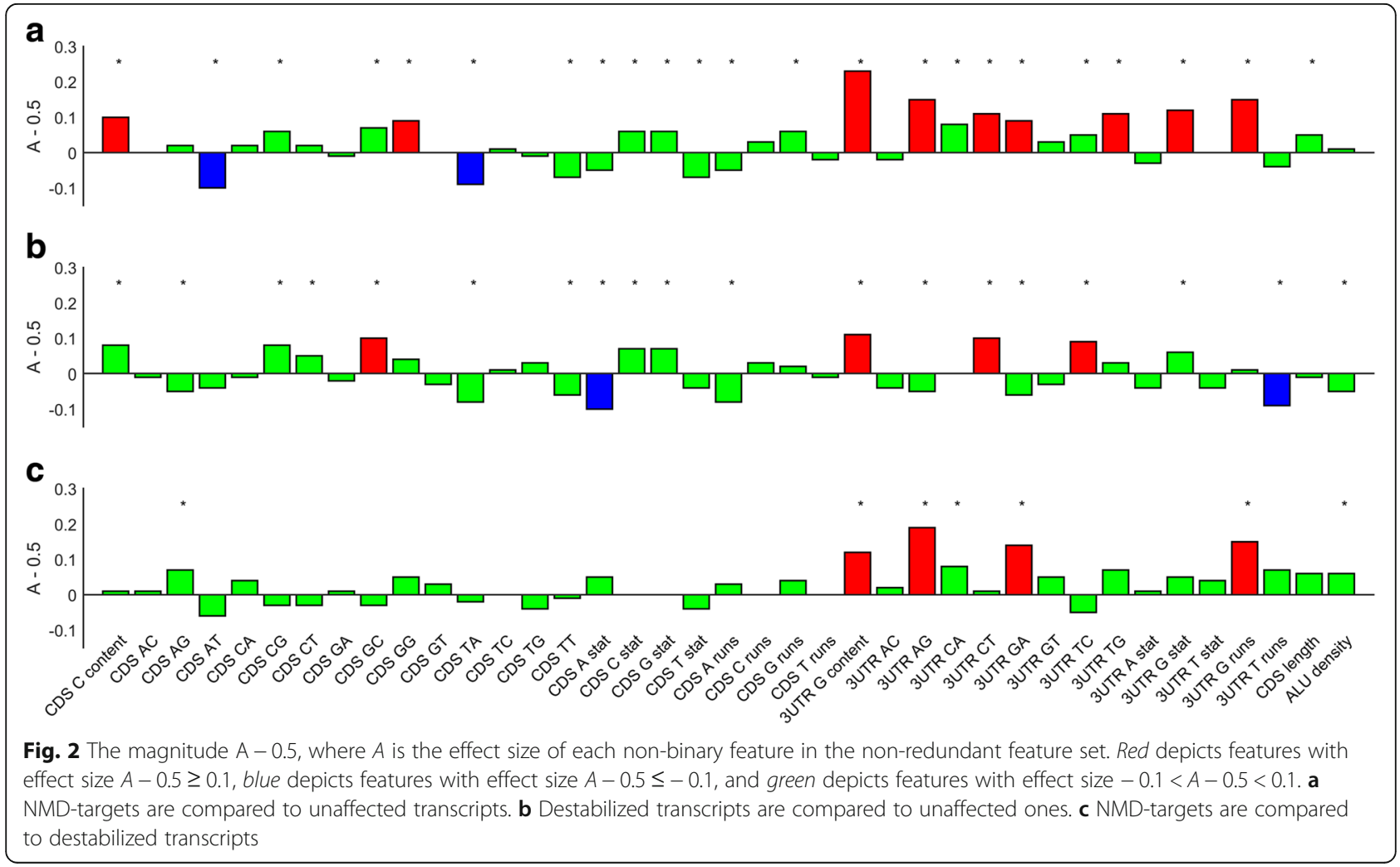



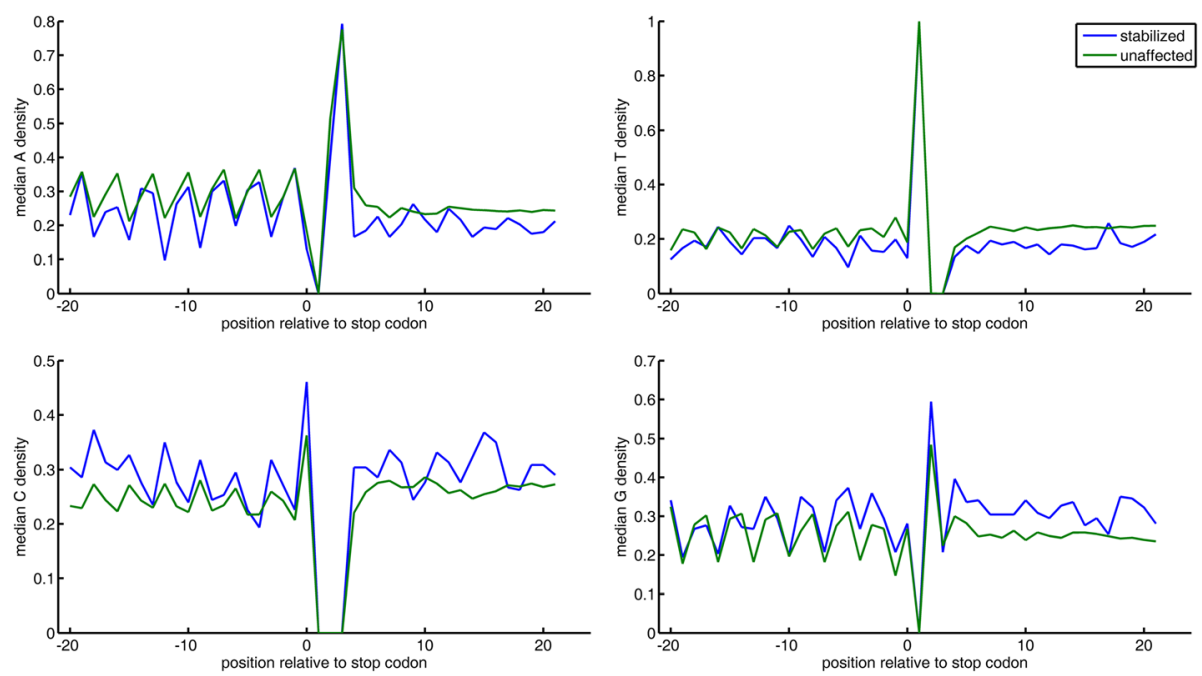

Fig. 3 Mononucleotide densities around the TC in stabilized and unaffected transcripts

S3), suggesting a strong depletion in destabilized transcripts. However, this effect is attributed to the shorter 3'UTR of destabilized transcripts, as the ALU density along their 3'UTR shows low effect size when compared to stabilized transcripts $(A-0.5=0.04)$ and to unaffected ones $(A-0.5=0.05)$.

Our results suggest that true NMD-targets are characterized by elevated G content around the TC, especially along the 3'UTR. To test the robustness of this conclusion, we have repeated the above analyses using the strict classification criterion. We observe qualitatively similar results (Additional file 2: Figures S1B, S2), although the smaller size of the stabilized and destabilized groups reduce the statistical power of the analysis. In addition, we wanted to account for the possible scenario that a transcript in our data set appears as lacking 3'UTR EJC in Refseq, and that another transcript that is indistinguishable by the BRIC-seq setup does harbor 3'UTR EJC but is absent in Refseq. To this end, we have examined all transcripts against the more permissive Ensembl database, and found that 178 out of the 217 NMD-targets (82\%) do not have any isoform with 3'UTR EJCs in Ensembl. Repeating the analysis above for the reduced set of these 178 NMD-targets led to qualitatively similar results (Additional file 2: Figure S3).

\section{More RNA secondary structures near the TC of NMD-targets}

Why high G content around the TC promotes NMD? We suggest that high $\mathrm{G}$ content around the TC increases the likelihood of the formation of secondary structures there, which hinders normal translation termination. Several examinations of codon usage bias towards the gene end had found a decrease in G in normally translated transcripts. Early works on E.coli genes revealed that they tend to end with AT-rich codons [53]. This was shortly followed with identical observations in $B$. subtilis [52] and yeast [54]. It has been previously shown that the presence of mRNA secondary structures can stall the ribosome during the elongation phase [60], or even lead to translation abortion [61]. Impeding the kinetics of translation termination may lead to a near cognate tRNA recognizing the termination codon as a sense codon and reads through it [62]. By this hypothesis, high $G$ content near the gene end promotes the formation of RNA secondary structures, which interfere with normal translation termination and activates NMD. Enrichment in G along the 3'UTR even in regions that are far from the TC may have other reasons, which will be detailed in the Discussion.

We therefore wanted to test whether stabilized transcripts are associated with increased stability of secondary structures around the TC. For this, we used RNAfold [63] to compute the minimum free energy (MFE) around the TC. Since MFE is negative, we used its absolute value so that higher values are indicative of more stable secondary structures. We found that MFE absolute values are significantly different between stabilized, unaffected, and destabilized transcripts upstream to the TC $\left(P=3.1 \cdot 10^{-9}\right.$, Kruskal-Wallis test $)$ and along the 3'UTR $\left(P=3.4 \cdot 10^{-6}\right.$, Kruskal-Wallis test). We then used Dunn's test to find which transcript groups significantly differ from each other (Table 1), and computed their corresponding nonparametric common language effect size. We found that stabilized transcripts have significantly higher MFE absolute value than unaffected transcripts both upstream to the $\mathrm{TC}$ and along the 3'UTR, whereas destabilized transcripts only differ from unaffected ones upstream to the TC. This result fully agrees with our inference from nucleotide composition, 
Table 1 Comparison of MFE absolute values between stabilized, unaffected, and destabilized transcripts, both upstream to the TC and along the $3^{\prime} U T R$

\begin{tabular}{|c|c|c|c|c|c|}
\hline \multicolumn{2}{|c|}{ Compared transcripts } & \multicolumn{3}{|c|}{ Dunn test } & \multirow{2}{*}{$\begin{array}{l}\text { Effect } \\
\text { size }\end{array}$} \\
\hline & & Q value & Critical Q & Decision & \\
\hline \multirow{2}{*}{$\begin{array}{l}\text { Stabilized vs. } \\
\text { unaffected }\end{array}$} & Upstream to the TC & 5.45 & 2.38 & Reject $H_{0}$ & 0.11 \\
\hline & Along the $3^{\prime} \cup T R$ & 4.71 & 2.38 & Reject $H_{0}$ & 0.09 \\
\hline \multirow{2}{*}{$\begin{array}{l}\text { Stabilized vs. } \\
\text { destabilized }\end{array}$} & Upstream to the TC & 1.17 & 2.38 & Fail to reject $H_{0}$ & 0.03 \\
\hline & Along the $3^{\prime} U T R$ & 4.36 & 2.38 & Reject $H_{0}$ & 0.14 \\
\hline \multirow{2}{*}{$\begin{array}{l}\text { Destabilized vs. } \\
\text { unaffected }\end{array}$} & Upstream to the TC & 3.30 & 2.38 & Reject $H_{0}$ & 0.07 \\
\hline & Along the $3^{\prime} \cup T R$ & 2.28 & 2.38 & Fail to reject $H_{0}$ & 0.05 \\
\hline
\end{tabular}

$H_{0}$ represents the null hypothesis of no difference between the two transcript groups. Effect size is defined as $|A-0.5|$, where $A$ is the common language effect size

and suggests that NMD-targets harbor a higher density of stable RNA secondary structures around the TC and, especially, along the 3'UTR.

In order to make the above analysis more quantitative, we wished to use a measure for the level of stability change of a transcript upon UPF1 knockdown, and to test whether it is correlated with the absolute value of MFE. To this end, we defined a measure for the stability change of a transcript (see Methods), and used it to divide the stabilized transcripts into three groups (small, moderate, and large stability change). We used KruskalWallis test to check whether these groups are characterized by different absolute values of MFE, and found that while MFE absolute values upstream to the TC are not related to the strength of the stability change, those along the 3'UTR certainly do $\left(P=4.0 \cdot 10^{-3}\right.$, KruskalWallis test; Table 2). Moreover, transcripts with large stability change are characterized by significantly more stable RNA secondary structures along their 3'UTR than transcripts with small and moderate stability change. Although transcripts with moderate stability change show statistically indistinguishable MFE absolute values than those of small stability change, they are nevertheless slightly higher (Table 2). Similarly, while the three groups of transcripts show statistically identical MFE absolute values upstream of the $\mathrm{TC}$, there is still a clear trend of higher values related to larger stability change. These results suggest that higher densities of stable RNA secondary structures around the $\mathrm{TC}$ and particularly along the 3'UTR are associated with higher stability change of the transcript following UPF1 knockdown.

\section{Validating the model on independent NMD-target set}

To provide further support to our model, we had examined an independent set of experimentally validated EJCindependent NMD-targets. For this, we used a recent experiment by Schmidt et al. [64], who identified endogenous transcripts that are cleaved by SMG6 using Parallel Analysis of RNA Ends (PARE). These transcripts are potential NMD-targets, as cleavage by SMG6 is the initial step in NMD $[65,66]$. Overall, Schmidt et al. identified 418 transcripts that are potential NMDtargets. Of these, we identified 171 transcripts that lack 3'UTR EJCs and are therefore potential EJC-independent NMD-target (see Methods). We have used this set of transcripts as our validation set, as well as a subset of 51 transcripts that are also up-regulated upon knocking down UPF1, SMG6, or both. We compared these EJCindependent NMD-targets to a list of NMD-insensitive transcripts that we prepared by taking the canonical transcripts (i.e., those with the longest CDS) of all human genes (hg19 RefSeq annotations), excluding genes

Table 2 Comparison of MFE absolute values between transcripts with small, moderate, and large stability change, both upstream to the TC and along the $3^{\prime} U T R$

\begin{tabular}{|c|c|c|c|c|c|}
\hline \multirow[t]{2}{*}{ Compared transcripts } & & \multicolumn{3}{|c|}{ Dunn test } & \multirow{2}{*}{$\begin{array}{l}\text { Effect } \\
\text { size }\end{array}$} \\
\hline & & $\mathrm{Q}$ value & Critical Q & Decision & \\
\hline \multirow{2}{*}{$\begin{array}{l}\text { Large vs. small } \\
\text { stability change }\end{array}$} & Upstream to the TC & 0.55 & 2.38 & Fail to reject $H_{0}$ & 0.03 \\
\hline & Along the $3^{\prime} U T R$ & 3.29 & 2.38 & Reject $H_{0}$ & 0.15 \\
\hline \multirow{2}{*}{$\begin{array}{l}\text { Large vs. moderate } \\
\text { stability change }\end{array}$} & Upstream to the TC & N/A & 2.38 & $\mathrm{~N} / \mathrm{A}$ & 0.02 \\
\hline & Along the $3^{\prime} U T R$ & 2.03 & 2.38 & Fail to reject $H_{0}$ & 0.10 \\
\hline \multirow{2}{*}{$\begin{array}{l}\text { Moderate vs. small } \\
\text { stability change }\end{array}$} & Upstream to the TC & 0.17 & 2.38 & Fail to reject $H_{0}$ & 0.01 \\
\hline & Along the $3^{\prime} U T R$ & 1.23 & 2.38 & Fail to reject $H_{0}$ & 0.06 \\
\hline
\end{tabular}

$H_{0}$ represents the null hypothesis of no difference between the two transcript groups. Effect size is defined as $|A-0.5|$, where $A$ is the common language effect size 
that are reported as NMD-targets by Tani et al. or by Schmidt et al. (14,983 transcripts in total).

Analyzing this set of EJC-independent NMD-targets, we noticed that they tend to be short, both in the CDS and in the 3'UTR (Fig. 4; Additional file 1: Table S5), possibly suggesting previously unnoticed bias in the experiment. Due to their short 3'UTR, they are depleted in nucleotide runs, reflected by low values of the $\chi^{2}$-statistics. Reassuringly, this set of NMD-targets show very similar nucleotide composition to the one we observed for our set of NMD-targets, namely higher $C$ and particularly $\mathrm{G}$ contents upstream to the $\mathrm{TC}$ and throughout the 3'UTR (Additional file 1: Table S5).

\section{Discussion}

One of the challenging tasks in studying NMD is the genome-wide identification of its targets. Most studies equate NMD-targets with transcripts whose expression level increases following UPF1 knockdown [18-20]. However, the complex networks of protein and gene interactions lead to many indirect dependencies on UPF1 levels. As a result, transcripts that increase their expression level following UPF1 knockdown are not necessarily NMD-targets, and transcripts that are NMD-targets may seem insensitive to UPF1 knockdown [56]. In addition, expression levels show high variability among cell populations and across cell types, and the efficiency of NMD varies between cells and under different physiological conditions [67-70]. As a result, different studies identified only partially overlapping sets of NMD-targets. In order to reduce the fraction of misidentified transcripts, we have defined NMD-targets as transcripts whose mRNA half-life increases following UPF1 knockdown. While it is still plausible that some NMD-targets would be misidentified by this criterion, it is nonetheless a more direct approach, and less prone to secondary effects [71].

We have used Tani et al. data of mRNA half-life alteration following UPF1 knockdown [55], and developed our own technique to identify NMD-targets. It is important to reiterate what these targets really are. First, NMD is known to operate through EJC-dependent and EJC-independent pathways [32]. In order to be compatible with Tani et al.'s transcript nomenclature, we have used a transcript database (RefSeq) that is heavily depleted with 3'UTR EJC-bearing transcripts [19]. In total, among the 4715 transcripts that could be classified, only 14 harbor 3'UTR EJCs. Hence, the current study focuses on 3'UTR EJC-independent NMD-targets. Second, UPF1 is not critical only to NMD, but also to Staufenmediated mRNA decay (SMD) [72]. So what we call here NMD-targets are in fact transcripts that are degraded by either SMD or 3'UTR EJC-independent NMD. We assume, however, that NMD-targets form the majority of these degraded transcripts.

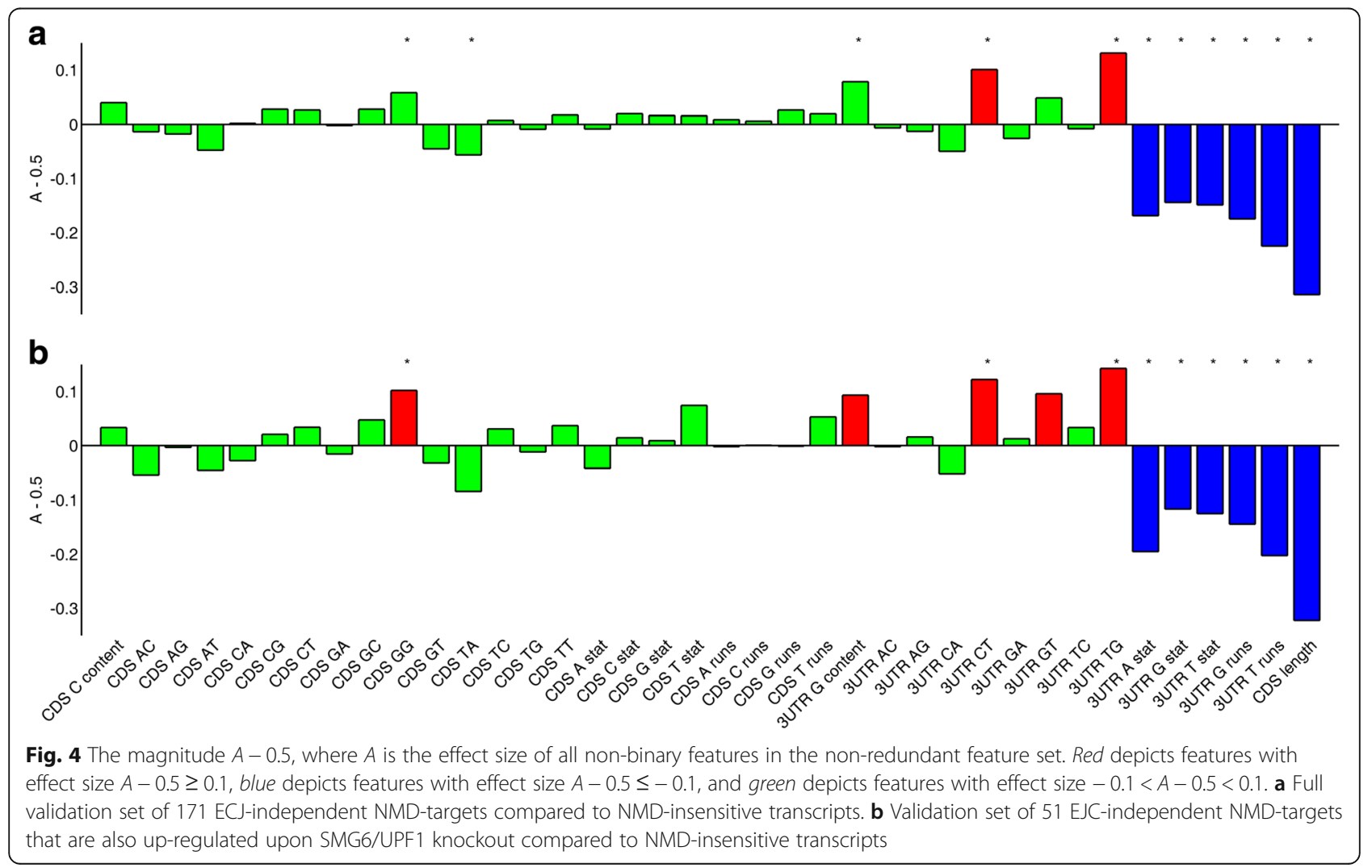


In addition to stabilized (NMD-target) and unaffected (NMD-insensitive) transcripts, we have identified a third group of transcripts that are destabilized upon UPF1 knockdown. Such transcripts are instable in the absence of NMD, possibly suggesting that their stability is maintained through the suppression of certain NMD-targets. It remains to be studied what is the mechanism that regulates the stability of this unique group of transcripts.

We found that EJC-independent NMD-targets have high G content upstream to their TC and throughout their 3'UTR. The most discriminative feature of NMD-targets is 3 'UTR G content $(A-0.5=0.23, P=$ $2.1 \cdot 10^{-28}$, Additional file 1: Table S4). While destabilized transcripts also display high 3'UTR G content $(A-0.5=$ $\left.0.11, P=3.4 \cdot 10^{-6}\right)$, it is still significantly lower than that of true NMD-targets $\left(A-0.5=0.12, P=3.8 \cdot 10^{-4}\right)$. This enrichment in $\mathrm{G}$ is also observed upstream to the TC of NMD-targets, although to a lesser extent than the enrichment along the 3'UTR. For example, the dinucleotide GG is enriched upstream of the TC in NMD-targets $(A-0.5=$ $\left.0.10 ; P=7.5 \cdot 10^{-6}\right)$, but not in destabilized transcripts $\left(A-0.5=0.04 ; P=9.6 \cdot 10^{-2}\right)$. This result was validated using an independent set of NMD-targets (Fig. 4, Additional file 1: Table S5). We showed that the enrichment of $\mathrm{G}$ nucleotides in NMD-targets results in higher density of stable RNA secondary structures around the TC (Table 1), and that NMD efficiency increases with the density of these secondary structures (Table 2). While the power of 3'UTR MFE to discriminate stabilized transcripts from unaffected ones $(A-0.5=0.09)$ is lower than that of 3'UTR G content $(\mathrm{A}-0.5=0.23)$, the density of 3'UTR MFE values (normalized to 3'UTR length) has a comparable discriminative power as the 3'UTR G content $(A-0.5=$ $0.22, P=1.1 \cdot 10^{-27}$ stabilized versus unaffected; $A-0.5=$ $0.11, P=2.5 \cdot 10^{-04}$ stabilized versus destabilized).

The effect of 3'UTR G content on NMD is probably not fully explained by the formation of secondary structures, as they may form quite far from the TC. There are other arguments suggesting that UPF1 activity is directly related to the G content along the 3'UTR. First, it was observed that UPF1 binding sites have high G content [20]. Second, UPF1 exhibits a helicase activity, and helicases are known to pauses at G-rich regions [43]. Therefore, Hurt et al. asserted that G-runs along the 3'UTR of NMD-targets block UPF1 scanning and lead to its accumulation at the 3'UTR [20]. However, this explanation may be challenged. First, it was shown that UPF1 binding is not an indicator of NMD activity, as both hyper-phosphorylated and hypo-phosphorylated UPF1 bind to the same sites, but it is only the hyperphosphorylated UPF1 that is relevant to NMD [44]. Second, the claim that UPF1 binding sites are G-rich was not replicated in a recent study by Zund et al.
[48]. We believe that an alternative model is also plausible, by which high G content along the 3'UTR increases the propensity of forming secondary structures, which promotes the recruitment of various RNA-binding proteins that may interfere in different ways with the translation termination complex. We believe that further studies should be conducted in order to determine the correct explanation.

Long 3'UTRs are believed to be enriched in NMDtargets $[38,39]$, presumably because they extend the physical distance between the TC and the poly(A) tail. It is therefore surprising that in our data 3'UTR length is similar between NMD-targets and NMDinsensitive transcripts $(A-0.5=0.04, P=0.06)$. Even more striking is our analysis of Schmidt et al.'s EJCindependent NMD-targets, which showed that they have significantly shorter 3'UTRs $(A-0.5=-0.27$, $\left.P=9.9 \cdot 10^{-19}\right)$. These observations suggest that EJCindependent NMD is independent of the 3'UTR length. Similarly, none of the other known NMDtriggering features (such as the presence of tuORFs) are enriched in our set of NMD-targets (Additional file 2: Figure S1). We therefore conclude that it is the nucleotide composition around the TC that mainly drives EJC-independent NMD.

While several other studies identified sets of putative NMD-targets, our focus on reliable EJC-independent NMD-targets made most of them irrelevant for the current study. For example, Andersen et al. [73] identified NMD-targets by knocking down SMG6, but all his identified NMD-targets harbor 3'UTR EJCs. Also, we avoided NMD-target sets that relied on expression data. The data produced by Schmidt et al. best suits our criteria. First, it avoids the use of expression to define NMD-targets. Instead, they were defined by examining SMG6 cleavage. Second, it is not exclusively made up of 3'UTR EJC-containing transcripts, and thus includes sufficient number of targets of the EJC-independent NMD pathway.

\section{Conclusions}

We found that transcript features traditionally associated with NMD, such as the length of the 3'UTR and the presence of tuORF, do not characterize EJCindependent NMD targets. Rather, EJC-independent NMD is likely triggered following certain nucleotide composition features that lead to elevated levels of RNA secondary structures. The main feature is high density of $G$ nucleotides upstream of the TC, and even more so along the 3'UTR. We propose that stable RNA secondary structures that are formed around the TC or along the 3'UTR interfere with normal translation termination leading to transcript instability and to the activation of NMD. 


\section{Methods}

\section{BRIC-seq analysis pipeline}

BRIC-seq is a method to determine RNA stability by monitoring the decrease of 5'-bromo-uridine-labeled (BrU-labeled) RNA [55, 74]. Tani et al. used BRICseq to measure RNA stability in two control and two UPF1-knockdown replicates by measuring the expression level of BrU-labeled RNA at four time points: $0,4,8$, and $12 \mathrm{~h}$ following the RNA pulse labeling [55]. We have downloaded their BRIC-seq raw data (accession numbers: DRA000591, DRP000622, DRS001594 - DRS001618, DRX001669 - DRX001693, and DRR002251 - DRR002275) from DDBJ (http:// www.ddbj.nig.ac.jp/), and followed their computational protocol of determining the normalized FPKM in each sample, as detailed in Imamachi et al. [74]. In brief, FASTQ files were filtered for low quality reads using the FASTX-Toolkits (http://hannonlab.cshl.edu/fastx_toolkit/index.html), rRNA-derived reads were filtered out via "Bowtie - un" command [75], and the remaining reads were mapped to the UCSC hg19 reference using the default settings of TopHat [75], and directed by Refseq transcripts annotation data (gtf format). Mapped reads (i.e., BAM files) were then assembled by Cufflinks [76], and the relative expression levels (FPKM) for Refseq transcripts were calculated. As recommended for such analyses [55, 74, 77], relative expression levels were scaled to that of a stable transcript (GAPDH gene) $[55,74]$. These normalization steps were carried out using a Perl script obtained from Imamachi et al. [74].

\section{Classifying transcripts by their response to UPF1-knockdown} By the experimental setup of Tani et al., the RNA abundance of any transcript should not increase with time. To filter out transcripts in which this is not the case, we have defined $\tau_{i, t}=\frac{1}{2}\left(c_{i, t}^{1}+c_{i, t}^{2}-c_{i, t=4}^{1}-c_{i, t=4}^{2}\right)$ to be the mean difference between the normalized FPKM control level of transcript $i$ at time $t \in\{8 h, 12 h\}$ and at time $t=4 \mathrm{~h}$. Similarly, we have defined $\varphi_{i, t}=\frac{1}{2}$ $\left(k_{i, t}^{1}+k_{i, t}^{2}-k_{i, t=4}^{1}-k_{i, t=4}^{2}\right)$ to be the mean difference between the normalized FPKM UPF1-knockdown level of transcript $i$ at time $t \in\{8 h, 12 h\}$ and at time $t=4 h$. We cannot make a proper hypothesis testing using only two biological replicates, but as an approximation we can treat $\bar{c}_{i, t}=\frac{1}{2}\left(c_{i, t}^{1}+c_{i, t}^{2}\right)$ as a mean value with standard error $\Delta \bar{c}_{i t} \approx \frac{1}{\sqrt{2}}\left|c_{i, t}^{1}-c_{i, t}^{2}\right|$, and then by means of error propagation,

$$
\Delta \tau_{i, t} \approx \frac{1}{\sqrt{2}} \sqrt{\left(c_{i, t}^{1}-c_{i, t}^{2}\right)^{2}+\left(c_{i, t=4 h}^{1}-c_{i, t=4 h}^{2}\right)^{2}} .
$$

Similarly, $\Delta \bar{k}_{i, t} \approx \frac{1}{\sqrt{2}}\left|k_{i, t}^{1}-k_{i, t}^{2}\right|$ and

$$
\Delta \varphi_{i, t} \approx \frac{1}{\sqrt{2}} \sqrt{\left(k_{i, t}^{1}-k_{i, t}^{2}\right)^{2}+\left(k_{i, t=4 h}^{1}-k_{i, t=4 h}^{2}\right)^{2}} .
$$

Using these expressions, we have filtered out transcripts in which the control mRNA abundance does not decay with time. To this end, we have used the FDRcorrected $p$-values $p \tau_{i, t}=1-\Phi\left(\tau_{i, t} / \Delta \tau_{i, t}\right)$, where $\Phi(x)$ is the cumulative standard normal distribution function, and removed all transcripts for which $p \tau_{i, t}<0.05$ for any of $t \in\{8 h, 12 h\}$. We have repeated this procedure for transcripts in which the UPF1-knockdown samples do not seem to decay with time, using the FDRcorrected $p$-values $p \phi_{i, t}=1-\Phi\left(\phi_{i, t} / \Delta \phi_{i, t}\right)$ (Additional file 2: Figure $S 4)$. Overall, this process led to the removal of 11 transcripts.

Next, we wished to classify the remaining transcripts according to their response to UPF1-knockdown. To this end, we have defined $\delta_{i, t}=\frac{1}{2}\left(c_{i, t}^{1}+c_{i, t}^{2}-k_{i, t}^{1}-k_{i, t}^{2}\right)$ as the mean difference of the normalized FPKM level of transcript $i$ between the control and the UPF1knockdown samples at time $t$. By means of error propagation,

$$
\Delta \delta_{i, t} \approx \frac{1}{\sqrt{2}} \sqrt{\left(c_{i, t}^{1}-c_{i, t}^{2}\right)^{2}+\left(k_{i, t}^{1}-k_{i, t}^{2}\right)^{2}} .
$$

We defined a transcript as stabilized at time $t$ if its mRNA abundance after UPF1-knockdown was significantly higher than its abundance in the control, using the FDR-corrected $p$-value $p \delta_{i, t}=\Phi\left(\delta_{i, t} / \Delta \delta_{i, t}\right)$. Likewise, we defined a transcript as destabilized at time $t$ if its mRNA abundance after UPF1-knockdown was significantly lower than its abundance in the control, using the FDR-corrected $p$-value $\tilde{p} \delta_{i, t}=1-\Phi\left(\delta_{i, t} / \Delta \delta_{i, t}\right)$ (Additional file 2: Figure S4).

Using these definitions, we have devised two criteria to classify transcripts into stabilized, destabilized, and unaffected. The standard criterion is the one that we use throughout the paper, and it looks at the mRNA abundance at times $t=8 \mathrm{~h}$ and $t=12 \mathrm{~h}$. Specifically, the classification scheme is (Fig. 1):

- A transcript is called stabilized (NMD-target) if $p \delta_{i, t} \leq 0.05$, and also $\min \left(k_{i, t}^{1}, k_{i, t}^{2}\right)>\max \left(c_{i, t}^{1}, c_{i, t}^{2}\right)$ for $t=8 h, 12 h$ (meaning, all replicates of the UPF1knockdown samples are above all replicates of the control samples for times $8 h$ and $12 h$ ).

- A transcript is called destabilized if $\tilde{p} \delta_{i, t} \leq 0.05$, and also $\min \left(c_{i, t}^{1}, c_{i, t}^{2}\right)>\max \left(k_{i, t}^{1}, k_{i, t}^{2}\right)$ for $\mathrm{t}=8 h, 12 h$.

- A transcript is called unaffected if both $p \delta_{i, t}>0.05$ and $\tilde{p} \delta_{i, t}>0.05$ for $t=8 \mathrm{~h}, 12 \mathrm{~h}$. 
Notably, some transcripts do not fall into any of these classes, and are consequently marked as unclassified (Additional file 1: Table S1). To test the robustness of our classification scheme, we employed a second, stricter, criterion that looks at the mRNA abundance also at $t=4 h$. Specifically:

- A transcript is called stabilized (NMD-target) if $p \delta_{i, t} \leq 0.05$, and also $\min \left(k_{i, t}^{1}, k_{i, t}^{2}\right)>\max \left(c_{i, t}^{1}, c_{i, t}^{2}\right)$ for $t=4 h, 8 h, 12 h$.

- A transcript is called destabilized if $\tilde{p} \delta_{i, t} \leq 0.05$, and also $\min \left(c_{i, t}^{1}, c_{i, t}^{2}\right)>\max \left(k_{i, t}^{1}, k_{i, t}^{2}\right)$ for $t=4 h, 8 h, 12 h$.

- A transcript is called unaffected if both $p \delta_{i, t}>0.05$ and $\tilde{p} \delta_{i, t}>0.05$ for $t=4 h, 8 h, 12 h$.

This stricter classification scheme resulted in more unclassified transcripts (Additional file 1: Table S1).

\section{Measuring stability change}

The stability change of transcript $i, S_{i}$, is defined as the median of $\delta_{\mathrm{i}, \mathrm{t}}$ at times $t=4 \mathrm{~h}, 8 \mathrm{~h}, 12 \mathrm{~h}$. We split stabilized transcripts to three groups based on their stability change value: small activity change (lower than or equal to the $33^{\text {rd }}$ percentile), moderate activity change (from $33^{\text {rd }}$ to $66^{\text {th }}$ percentiles), and large activity change (higher than or equal to the $66^{\text {th }}$ percentile).

\section{Generating a set of non-redundant features}

In order to remove highly correlated features, we first ranked all the non-binary features based on the nonparametric common language effect size (A), measuring for each feature how strongly it differs between stabilized and the unaffected transcripts. Then, from each pair of highly-correlated features $(a b s(R) \geq 0.7$, where $R$ is the Spearman correlation coefficient), we recursively removed the lower ranked one. The four binary features were automatically included in the non-redundant set. At the end of the process we were left with 43 nonredundant features (Additional file 1: Table S2).

When looking at tuORFs, we have made a distinction between transcripts in which the tuORF is strictly within the 5'UTR, and those in which the tuORF overlaps the main ORF. The former may repress decay due to translation re-initiation at the main ORF, while the latter wouldn't allow this.

\section{Non-parametric common language effect size}

For a feature $x$ measured for samples coming from two classes $C_{1}$ and $C_{2}$, the effect size $A$ is defined as the probability that the feature value of a random sample from $C_{1}$ is higher than its value for a random sample from $C_{2}$ [59]. Let $n_{1}$ and $n_{2}$ be the number of samples in classes $C_{1}$ and $C_{2}$, respectively. Let us look at all pairs of samples such that one is from $C_{1}$ and the other is from
$C_{2}$. From among these pairs, let $n_{C_{1}>C_{2}}$ be the number of pairs in which $x\left(C_{1}\right)>x\left(C_{2}\right)$. Similarly, let $n_{C_{1}=C_{2}}$ be the number of pairs in which $x\left(C_{1}\right)=x\left(C_{2}\right)$. Then, the effect size $A$ is calculated by

$$
A=\frac{n_{C_{1}>C_{2}}+\frac{1}{2} n_{C_{1}=C_{2}}}{n_{1} n_{2}} .
$$

\section{Runs of nucleotides and the $x^{2}$-statistic}

For every sequence $i$ and nucleotide of type $s$, the probability $p$ of having a run of exactly $m$ consecutive appearances of $s$ is

$$
p_{m, s, i}=\delta_{s, i}{ }^{m} \cdot\left(1-\delta_{s, i}\right)^{2},
$$

where $\delta_{s, i}$ is the density of nucleotide $s$ in sequence $i$. If the length of sequence $i, l_{i}$, is sufficiently long, the expected number of runs is approximately

$$
E_{m, s, i}=p_{m, s, i} \cdot l_{i} \text {. }
$$

Let $O_{m, s, i}$ be the observed number of runs of $m$ consecutive appearances of nucleotide $s$ in sequence $i$. In practice, we shall be interested in runs of minimum length $m=3$, and up to the practical limit of length $m=$ 7. Based on the goodness of fit test, the $\chi^{2}$-statistic for sequence $i$ and for nucleotide $s$ is given by

$$
\chi_{s, i}^{2}=\sum_{m=3}^{7} \frac{\left(\sqrt{\frac{n_{O}}{n_{E}} \cdot E_{m, s, i}}-\sqrt{\frac{n_{E}}{n_{O}} \cdot O_{m, s, i}}\right)^{2}}{E_{m, s, i}+O_{m, s, i}} .
$$

Here, $n_{O}=\sum_{m=3}^{7} O_{m, s, i}$ is the total number of observed $s$-runs, and $n_{E}=\sum_{m=3}^{7} E_{m, s, i}$ is the total number of expected $s$-runs.

\section{Ribosome footprint data}

Data on tuORFs were taken from https://www.ncbi.nlm.nih.gov/pmc/articles/PMC3483550.

\section{Predicting RNA secondary structures}

We used the default parameters of RNAfold 2.1.8 [63] from the Vienna Package to predict local stable secondary structures. The minimum free energy (MFE) was computed and its absolute value was used throughout the study.

\section{Construction of the validation set}

We used BioMart to convert Schmidt et al.'s transcript names [64] to Ensembl IDs, ending with a successful conversion of 327 out of the original 418 NMD-targets. We removed transcripts with more than one corresponding Ensembl ID, as well as transcripts that lack 3'UTR or CDS annotations.

\section{Additional files}

Additional file 1: Table S1. Summary of transcript classification based on how their mRNA abundance decay curve is affected by UPF1-knockdown. Table S2. Putative NMD-triggering features examined in the current study. a Features marked by 'Yes' belong to the non-redundant set, meaning that 
they are sufficiently uncorrelated to each other. b Features marked by 'Yes' are significantly different between NMD-targets and unaffected transcripts $(P<0.05$, FDR-corrected $U$-test) and also have high effect size $(|A-0.5| \geq 0.1)$. c Features marked by 'Yes' are significantly different between destabilized and unaffected transcripts $(P<0.05$, FDR-corrected $U$-test) and also have high effect size $(|A-0.5| \geq 0.1)$. Table S3. The level of enrichment of each binary feature (its fraction in one class divided by its fraction in the second class). Asterisks denote significant enrichments ( $P<0.05$, Fisher exact test). Table S4. Effect size (ES, $A-0.5)$ and $P$-value (FDR-corrected, $U$-test) of the non-redundant features, for all pairwise comparisons of transcript groups. Table S5. Effect size (ES, $A-0.5)$ and $p$-value (FDR-corrected, $U$-test) of the non-redundant features for the two validation sets. (PDF $242 \mathrm{~kb}$ )

Additional file 2: Figure S1. For each binary feature (x-axis) and transcript group (colors), the bars show the percentage of transcripts in which the feature is present. Error bars indicate one binomial standard deviation. (A) The transcripts were classified by the standard classification scheme. (B) The transcripts were classified by the strict classification scheme. Figure S2. The magnitude $A-0.5$, where $A$ is the effect size of each non-binary feature in the non-redundant feature set, and where the transcripts were classified using the strict criterion. Red depicts features with effect size $A-0.5 \geq 0.1$, blue depicts features with effect size $A-$ $0.5 \leq-0.1$, and green depicts features with effect size $-0.1<A-0.5<$ 0.1. (A) NMD-targets are compared to unaffected transcripts. (B) Destabilized transcripts are compared to unaffected ones. (C) NMD-targets are compared to destabilized transcripts. Figure S3. NMD-targets are compared to unaffected transcripts, when only transcripts that do not have isoforms harboring 3'UTR EJC were taken into account. The bars show The magnitude $A-0.5$, where $A$ is the effect size of each non-binary feature in the non-redundant feature set. Red depicts features with effect size $A-0.5 \geq 0.1$, blue depicts features with effect size $A-0.5 \leq-0.1$, and green depicts features with effect size $-0.1<A-0.5<0.1$. Figure S4. A schematic description of the different parameters used to classify transcripts into stabilized, unaffected, and destabilized. (PDF $231 \mathrm{~kb}$ )

\section{Abbreviations}

CDS: Coding sequence; EJC: Exon-junction complex; MFE: Minimum free energy; NMD: Nonsense-mediated decay; PTC: Premature termination codon; TC: Termination codon; tuORF: Translated upstream open reading frame; uORF: Upstream open reading frame

\section{Acknowledgements}

We would like to thank Lynne Maquat for critical and useful comments on the manuscript.

\section{Funding}

This research was supported by the Israel Science Foundation (grant No. 1431/13).

\section{Availability of data and materials}

The datasets supporting the conclusions of this article were published by other authors, and are all freely available.

\section{Authors' contributions}

FZ analyzed the data and performed the work. LC conceived and supervised the study. Both authors read and approved the final manuscript.

\section{Competing interests}

The authors declare that they have no competing interests.

\section{Consent for publication}

Not applicable.

\section{Ethics approval and consent to participate}

Not applicable.

\section{Author details}

'Department of Genetics, The Alexander Silberman Institute of Life Sciences, Faculty of Science, The Hebrew University of Jerusalem, Edmond J. Safra Campus, Givat Ram, Jerusalem 91904, Israel. ${ }^{2}$ Hereditary Research Lab, Life Sciences Department, Bethlehem University, Bethlehem, Palestine.
Received: 17 June 2016 Accepted: 26 November 2016

Published online: 07 December 2016

\section{References}

1. Chang YF, Imam JS, Wilkinson MF. The nonsense-mediated decay RNA surveillance pathway. Annu Rev Biochem. 2007;76:51-74.

2. Silva AL, Romao L. The mammalian nonsense-mediated mRNA decay pathway: to decay or not to decay! Which players make the decision? FEBS Lett. 2009:583(3):499-505.

3. Schweingruber C, Rufener SC, Zund D, Yamashita A, Muhlemann O. Nonsense-mediated mRNA decay - mechanisms of substrate mRNA recognition and degradation in mammalian cells. Biochim Biophys Acta. 2013:1829(6-7):612-23.

4. Kervestin S, Jacobson A. NMD: a multifaceted response to premature translational termination. Nat Rev Mol Cell Biol. 2012:13(11):700-12.

5. Popp MW, Maquat LE. Organizing principles of mammalian nonsensemediated mRNA decay. Annu Rev Genet. 2013;47:139-65.

6. McGlincy NJ, Smith CW. Alternative splicing resulting in nonsense-mediated mRNA decay: what is the meaning of nonsense? Trends Biochem Sci. 2008; 33(8):385-93.

7. Wollerton MC, Gooding C, Wagner EJ, Garcia-Blanco MA, Smith CW. Autoregulation of polypyrimidine tract binding protein by alternative splicing leading to nonsense-mediated decay. Mol Cell. 2004;13(1):91-100.

8. Lewis BP, Green RE, Brenner SE. Evidence for the widespread coupling of alternative splicing and nonsense-mediated mRNA decay in humans. Proc Natl Acad Sci U S A. 2003:100(1):189-92.

9. Ge Y, Porse BT. The functional consequences of intron retention: alternative splicing coupled to NMD as a regulator of gene expression. BioEssays. 2014;36(3):236-43.

10. Holbrook JA, Neu-Yilik G, Hentze MW, Kulozik AE. Nonsense-mediated decay approaches the clinic. Nat Genet. 2004;36(8):801-8.

11. Frischmeyer PA, Dietz HC. Nonsense-mediated mRNA decay in health and disease. Hum Mol Genet. 1999:8(10):1893-900.

12. Khajavi M, Inoue K, Lupski JR. Nonsense-mediated mRNA decay modulates clinical outcome of genetic disease. Eur J Hum Genet. 2006;14(10):1074-81.

13. Nagy E, Maquat LE. A rule for termination-codon position within introncontaining genes: when nonsense affects RNA abundance. Trends Biochem Sci. 1998;23(6):198-9.

14. Cheng J, Belgrader P, Zhou X, Maquat LE. Introns are cis effectors of the nonsense-codon-mediated reduction in nuclear mRNA abundance. Mol Cell Biol. 1994;14(9):6317-25.

15. Sauliere J, Murigneux V, Wang Z, Marquenet E, Barbosa I, Le Tonqueze O, Audic Y, Paillard L, Roest Crollius H, Le Hir H. CLIP-seq of elF4Alll reveals transcriptome-wide mapping of the human exon junction complex. Nat Struct Mol Biol. 2012;19(11):1124-31.

16. Singh G, Kucukural A, Cenik C, Leszyk JD, Shaffer SA, Weng Z, Moore MJ. The cellular EJC interactome reveals higher-order mRNP structure and an EJC-SR protein nexus. Cell. 2012:151(4):750-64.

17. Maquat LE, Tarn WY, Isken O. The pioneer round of translation: features and functions. Cell. 2010;142(3):368-74.

18. Mendell JT, Sharifi NA, Meyers JL, Martinez-Murillo F, Dietz HC. Nonsense surveillance regulates expression of diverse classes of mammalian transcripts and mutes genomic noise. Nat Genet. 2004;36(10):1073-8.

19. Yepiskoposyan $\mathrm{H}$, Aeschimann F, Nilsson D, Okoniewski M, Muhlemann O Autoregulation of the nonsense-mediated mRNA decay pathway in human cells. RNA. 2011;17(12):2108-18

20. Hurt JA, Robertson AD, Burge CB. Global analyses of UPF1 binding and function reveal expanded scope of nonsense-mediated mRNA decay. Genome Res. 2013:23(10):1636-50.

21. Ramani AK, Nelson AC, Kapranov P, Bell I, Gingeras TR, Fraser AG. High resolution transcriptome maps for wild-type and nonsense-mediated decaydefective Caenorhabditis elegans. Genome Biol. 2009;10(9):R101.

22. Decourty L, Doyen A, Malabat C, Frachon E, Rispal D, Seraphin B, Feuerbach F, Jacquier A, Saveanu C. Long open reading frame transcripts escape nonsense-mediated mRNA decay in yeast. Cell Rep. 2014;6(4):593-8.

23. Hansen KD, Lareau LF, Blanchette M, Green RE, Meng Q, Rehwinkel J, Gallusser FL, Izaurralde E, Rio DC, Dudoit S, et al. Genome-wide identification of alternative splice forms down-regulated by nonsensemediated mRNA decay in Drosophila. PLoS Genet. 2009;5(6):e1000525

24. Wang J, Gudikote JP, Olivas OR, Wilkinson MF. Boundary-independent polar nonsense-mediated decay. EMBO Rep. 2002;3(3):274-9. 
25. Chan D, Weng YM, Graham HK, Sillence DO, Bateman JF. A nonsense mutation in the carboxyl-terminal domain of type $X$ collagen causes haploinsufficiency in schmid metaphyseal chondrodysplasia. J Clin Invest. 1998;101(7):1490-9.

26. Rajavel KS, Neufeld EF. Nonsense-mediated decay of human HEXA mRNA. Mol Cell Biol. 2001;21(16):5512-9.

27. Matsuda D, Hosoda N, Kim YK, Maquat LE. Failsafe nonsense-mediated mRNA decay does not detectably target elF4E-bound mRNA. Nat Struct Mol Biol. 2007;14(10):974-9.

28. Asselta R, Duga S, Spena S, Santagostino E, Peyvandi F, Piseddu G, Targhetta R, Malcovati M, Mannucci PM, Tenchini ML. Congenital afibrinogenemia: mutations leading to premature termination codons in fibrinogen A alpha-chain gene are not associated with the decay of the mutant mRNAs. Blood. 2001;98(13):3685-92.

29. Neerman-Arbez M, Germanos-Haddad M, Tzanidakis K, Vu D, Deutsch S, David A, Morris MA, de Moerloose P. Expression and analysis of a split premature termination codon in FGG responsible for congenita afibrinogenemia: escape from RNA surveillance mechanisms in transfected cells. Blood. 2004;104(12):3618-23.

30. Denecke J, Kranz C, Kemming D, Koch HG, Marquardt T. An activated 5' cryptic splice site in the human ALG3 gene generates a premature termination codon insensitive to nonsense-mediated mRNA decay in a new case of congenital disorder of glycosylation type Id (CDG-Id). Hum Mutat. 2004;23(5):477-86.

31. Stockklausner C, Breit S, Neu-Yilik G, Echner N, Hentze MW, Kulozik AE, Gehring NH. The uORF-containing thrombopoietin mRNA escapes nonsense-mediated decay (NMD). Nucleic Acids Res. 2006;34(8):2355-63.

32. Metze S, Herzog VA, Ruepp MD, Muhlemann O. Comparison of EJCenhanced and EJC-independent NMD in human cells reveals two partially redundant degradation pathways. RNA. 2013;19(10):1432-48.

33. Amrani N, Ganesan R, Kervestin S, Mangus DA, Ghosh S, Jacobson A. A faux 3'-UTR promotes aberrant termination and triggers nonsense-mediated mRNA decay. Nature. 2004;432(7013):112-8.

34. Silva AL, Ribeiro P, Inacio A, Liebhaber SA, Romao L. Proximity of the poly(A)-binding protein to a premature termination codon inhibits mammalian nonsense-mediated mRNA decay. RNA. 2008;14(3):563-76.

35. Behm-Ansmant I, Gatfield D, Rehwinkel J, Hilgers V, Izaurralde E. A conserved role for cytoplasmic poly(A)-binding protein 1 (PABPC1) in nonsense-mediated mRNA decay. EMBO J. 2007;26(6):1591-601.

36. Singh G, Rebbapragada I, Lykke-Andersen J. A competition between stimulators and antagonists of Upf complex recruitment governs human nonsense-mediated mRNA decay. PLoS Biol. 2008;6(4):e111.

37. Eberle AB, Stalder L, Mathys H, Orozco RZ, Muhlemann O. Posttranscriptional gene regulation by spatial rearrangement of the 3 untranslated region. PLoS Biol. 2008;6(4):e92.

38. Buhler M, Steiner S, Mohn F, Paillusson A, Muhlemann O. EJC-independent degradation of nonsense immunoglobulin-mu mRNA depends on 3' UTR length. Nat Struct Mol Biol. 2006;13(5):462-4.

39. Muhlrad D, Parker R. Aberrant mRNAs with extended $3^{\prime}$ UTRs are substrates for rapid degradation by mRNA surveillance. RNA. 1999;5(10):1299-307.

40. Toma KG, Rebbapragada I, Durand S, Lykke-Andersen J. Identification of elements in human long 3 ' UTRs that inhibit nonsense-mediated decay. Rna. 2015;21(5):887-97.

41. Wang W, Czaplinski K, Rao Y, Peltz SW. The role of Upf proteins in modulating the translation read-through of nonsense-containing transcripts. EMBO J. 2001;20(4):880-90.

42. Kashima I, Yamashita A, Izumi N, Kataoka N, Morishita R, Hoshino S, Ohno M, Dreyfuss G, Ohno S. Binding of a novel SMG-1-Upf1-eRF1eRF3 complex (SURF) to the exon junction complex triggers Upf1 phosphorylation and nonsense-mediated mRNA decay. Genes Dev. 2006:20(3):355-67.

43. Bhattacharya A, Czaplinski K, Trifillis P, He F, Jacobson A, Peltz SW. Characterization of the biochemical properties of the human Upf1 gene product that is involved in nonsense-mediated mRNA decay. RNA. 2000; 6(9):1226-35.

44. Kurosaki T, Li W, Hoque M, Popp MW, Ermolenko DN, Tian B, Maquat LE. A post-translational regulatory switch on UPF1 controls targeted mRNA degradation. Genes Dev. 2014;28(17):1900-16.

45. Kurosaki T, Maquat LE. Rules that govern UPF1 binding to mRNA $3^{\prime}$ UTRs. Proc Natl Acad Sci U S A. 2013;110:3357-62.
46. Lee SR, Pratt GA, Martinez FJ, Yeo GW, Lykke-Andersen J. Target Discrimination in Nonsense-Mediated mRNA Decay Requires Upf1 ATPase Activity. Mol Cell. 2015;59(3):413-25.

47. Hogg JR, Goff SP. Upf1 senses 3'UTR length to potentiate mRNA decay. Cell. 2010;143(3):379-89.

48. Zund D, Gruber AR, Zavolan M, Muhlemann O. Translation-dependent displacement of UPF1 from coding sequences causes its enrichment in $3^{\prime}$ UTRs. Nat Struct Mol Biol. 2013;20(8):936-43.

49. Johns L, Grimson A, Kuchma SL, Newman CL, Anderson P. Caenorhabditis elegans SMG-2 selectively marks mRNAs containing premature translation termination codons. Mol Cell Biol. 2007;27(16):5630-8.

50. Fatscher T, Boehm V, Gehring NH. Mechanism, factors, and physiological role of nonsense-mediated mRNA decay. Cell Mol Life Sci. 2015;72(23):4523-44.

51. Zund D, Muhlemann O. Recent transcriptome-wide mapping of UPF1 binding sites reveals evidence for its recruitment to mRNA before translation. Translation. 2013;1(2):e26977.

52. Rocha EP, Danchin A, Viari A. Translation in Bacillus subtilis: roles and trends of initiation and termination, insights from a genome analysis. Nucleic Acids Res. 1999;27(17):3567-76.

53. Eyre-Walker A. The close proximity of Escherichia coli genes: consequences for stop codon and synonymous codon use. J Mol Evol. 1996;42(2):73-8.

54. Katz $L$, Burge CB. Widespread selection for local RNA secondary structure in coding regions of bacterial genes. Genome Res. 2003;13(9):2042-51.

55. Tani H, Imamachi N, Salam KA, Mizutani R, ljiri K, Irie T, Yada T, Suzuki Y, Akimitsu N. Identification of hundreds of novel UPF1 target transcripts by direct determination of whole transcriptome stability. RNA Biol. 2012;9(11):1370-9.

56. Chapin A, Hu H, Rynearson SG, Hollien J, Yandell M, Metzstein MM. In vivo determination of direct targets of the nonsense-mediated decay pathway in Drosophila. G3. 2014:4(3):485-96.

57. Trcek $T$, Sato $H$, Singer RH, Maquat LE. Temporal and spatial characterization of nonsense-mediated mRNA decay. Genes Dev. 2013;27(5):541-51.

58. Gong C, Tang Y, Maquat LE. mRNA-mRNA duplexes that autoelicit Staufen1mediated mRNA decay. Nat Struct Mol Biol. 2013;20(10):1214-20.

59. Ruscio J. A probability-based measure of effect size: robustness to base rates and other factors. Psychol Methods. 2008;13(1):19-30.

60. Plat T. RNA Structure and function. New York: Cold Spring Harbour Laboratory Press; 1998.

61. Carpousis AJ, Vanzo NF, Raynal LC. mRNA degradation. A tale of poly(A) and multiprotein machines. Trends Genet. 1999;15(1):24-8.

62. Tate WP, Mannering SA. Three, four or more: the translational stop signal at length. Mol Microbiol. 1996;21(2):213-9.

63. Lorenz R, Bernhart SH, Honer Zu Siederdissen C, Tafer H, Flamm C, Stadler PF, Hofacker IL. ViennaRNA Package 2.0. Algorithms Mol Biol. 2011;6:26.

64. Schmidt SA, Foley PL, Jeong DH, Rymarquis LA, Doyle F, Tenenbaum SA, Belasco JG, Green PJ. Identification of SMG6 cleavage sites and a preferred RNA cleavage motif by global analysis of endogenous NMD targets in human cells. Nucleic Acids Res. 2015;43(1):309-23.

65. Huntzinger E, Kashima I, Fauser M, Sauliere J, Izaurralde E. SMG6 is the catalytic endonuclease that cleaves mRNAs containing nonsense codons in metazoan. RNA. 2008;14(12):2609-17.

66. Eberle AB, Lykke-Andersen S, Muhlemann O, Jensen TH. SMG6 promotes endonucleolytic cleavage of nonsense mRNA in human cells. Nat Struct Mol Biol. 2009;16(1):49-55.

67. Popp MW, Maquat LE. Attenuation of nonsense-mediated mRNA decay facilitates the response to chemotherapeutics. Nat Commun. 2015;6:6632.

68. Lou CH, Shao A, Shum EY, Espinoza JL, Huang L, Karam R, Wilkinson MF. Posttranscriptional control of the stem cell and neurogenic programs by the nonsense-mediated RNA decay pathway. Cell Rep. 2014;6(4):748-64.

69. Karam R, Lou CH, Kroeger H, Huang L, Lin JH, Wilkinson MF. The unfolded protein response is shaped by the NMD pathway. EMBO Rep. 2015;16(5):599-609.

70. Gong C, Kim YK, Woeller CF, Tang Y, Maquat LE. SMD and NMD are competitive pathways that contribute to myogenesis: effects on PAX3 and myogenin mRNAs. Genes Dev. 2009;23(1):54-66.

71. Peccarelli M, Kebaara BW. Regulation of natural mRNAs by the nonsensemediated mRNA decay pathway. Eukaryot cell. 2014;13(9):1126-35.

72. Park E, Maquat LE. Staufen-mediated mRNA decay. Wiley interdiscip Rev RNA. 2013;4(4):423-35. 
73. Lykke-Andersen S, Chen Y, Ardal BR, Lilje B, Waage J, Sandelin A, Jensen TH. Human nonsense-mediated RNA decay initiates widely by endonucleolysis and targets snoRNA host genes. Genes Dev. 2014;28(22):2498-517.

74. Imamachi N, Tani H, Mizutani R, Imamura K, Irie T, Suzuki Y, Akimitsu N. BRIC-seq: a genome-wide approach for determining RNA stability in mammalian cells. Methods. 2014;67(1):55-63.

75. Langmead B, Trapnell C, Pop M, Salzberg SL. Ultrafast and memory-efficient alignment of short DNA sequences to the human genome. Genome Biol. 2009:10(3):R25.

76. Trapnell C, Williams BA, Pertea G, Mortazavi A, Kwan G, van Baren MJ, Salzberg SL, Wold BJ, Pachter L. Transcript assembly and quantification by RNA-Seq reveals unannotated transcripts and isoform switching during cell differentiation. Nat Biotechnol. 2010;28(5):511-5.

77. Sharova LV, Sharov AA, Nedorezov T, Piao Y, Shaik N, Ko MS. Database for mRNA half-life of 19977 genes obtained by DNA microarray analysis of pluripotent and differentiating mouse embryonic stem cells. DNA Res. 2009;16(1):45-58.

Submit your next manuscript to BioMed Central and we will help you at every step:

- We accept pre-submission inquiries

- Our selector tool helps you to find the most relevant journal

- We provide round the clock customer support

- Convenient online submission

- Thorough peer review

- Inclusion in PubMed and all major indexing services

- Maximum visibility for your research

Submit your manuscript at www.biomedcentral.com/submit
C) Biomed Central 